\title{
Coherent Control of Optically Generated and Detected Picosecond Surface Acoustic Phonons
}

\section{Symposium on Laser Spectroscopy}

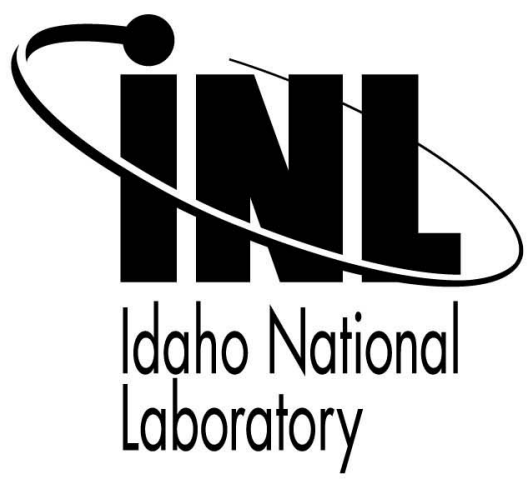

November 2006

This is a preprint of a paper intended for publication in a journal or proceedings. Since changes may be made before publication, this preprint should not be cited or reproduced without permission of the author. This document was prepared as an account of work sponsored by an agency of the United States Government. Neither the United States Government nor any agency thereof, or any of their employees, makes any warranty, expressed or implied, or assumes any legal liability or responsibility for any third party's use, or the results of such use, of any information, apparatus, product or process disclosed in this report, or represents that its use by such third party would not infringe privately owned rights. The views expressed in this paper are not necessarily those of the United States Government or the sponsoring agency. 


\title{
Coherent control of optically generated and detected picosecond surface acoustic phonons
}

\author{
David H. Hurley and Ryan Lewis \\ Idaho National Laboratory \\ Physics Department \\ Idaho Falls, ID 83415-2209, U.S.A.
}

\begin{abstract}
Coherent control of electronic and phononic excitations in solids, as well as chemical and biological systems on ultrafast time scales is of current research interest. In semiconductors, coherent control of phonons has been demonstrated for acoustic and optical phonons generated in superlattice structures. The bandwidth of these approaches is typically fully utilized by employing a 1-D geometry where the laser spot size is much larger than the superlattice repeat length. In this article we demonstrate coherent control of optically generated picosecond surface acoustic phonons using sub-optical wavelength absorption gratings. The generation and detection characteristics of two material systems are investigated (aluminum absorption gratings on Si and GaAs substrates). Constructive and complete destructive interference conditions are demonstrated using two pump pulses derived from a single Michelson interferometer.
\end{abstract}




\section{Introduction}

Surface acoustic phonons (SAP) have attracted a great deal of attention lately due to intriguing applications ranging from the modulation of electron properties of semiconductors to fundamental investigations of phonon mediated heat transport. A surface acoustic phonon is a particular acoustic mode that is confined to propagate near the surface of a sample. Hence, surface acoustic phonon experiments only require single side access, the source-receiver distance is dictated by the experimenter, and this acoustic mode geometrically attenuates less rapidly than bulk waves. These characteristics are particularly appealing for applications involving fundamental studies of phonon mediated heat transport ${ }^{1}$ and studies involving dynamic strain modulation of electronic properties of quantum structures..$^{2,3,4,5}$ For instance, coherent control of $\mathrm{MHz}$ frequency SAP has been used to influence decoherence of spin states in quantum well structures. ${ }^{4}$ Megahertz frequency SAP wave packets have also been used to drive single electron pumps $^{3}$ and to control recombination pathways. ${ }^{5}$ A common element in these approaches involves the use of interdigital elements for phonon generation. Large amplitude SAPs can be generated since the elements are electronically pumped at resonance. However, electrically driven interdigital transducers require a piezoelectrically active material for acoustic wave generation. For fundamental studies of phonon transport, this is a particularly inconvenient limitation since only a limited number of material systems and crystal orientations can be investigated.

Optical generation of picosecond SAP using suboptical wavelength lithographic absorption gratings provides an attractive alternative to piezoelectric generation ${ }^{6,7,8}$. The mechanism responsible for acoustic phonon generation involves a coupling between a laser-induced temperature gradient and strain, governed by lattice anharmonicity (thermoelasticity). The conditions placed on the substrate are removed since this acoustic phonon generation mechanism is present in all materials and along all directions. Furthermore, since ultrafast lasers are used for 
generation, the primary limitation on bandwidth is related to the inverse of the acoustic transit time across one period of the grating which is in turn related to the lithographic feature size. Using the (001) surface of silicon as an example and a feature size of $50 \mathrm{~nm}$ (a conservative estimate for electron beam lithography), the upper bandwidth limit is approximately $80 \mathrm{GHz}$.

\section{Experiments and Discussion}

To better understand this generation and detection scheme consider experiments involving aluminum gratings on GaAs and Si substrates. These samples were prepared using electron beam lithography. They consisted of $5 \mu \mathrm{m}$ long bars periodically deposited on (100) Si and (100) GaAs substrates. For the Si sample, the bar axis coincided with the [001] direction and for the GaAs sample the bar axis coincided with the [011] direction. The bar height, h, width, a, and periodicity, $\mathrm{p}$, are $50 \mathrm{~nm}, 110 \mathrm{~nm}$ and $220 \mathrm{~nm}$ respectively. The experimental setup is shown in

Fig. 1. The pump and probe pulse trains are derived from the same mode locked Ti:sapphire laser with $\sim 1$ ps pulse duration, and a pulse energy of .06 nJ. A shaker operated at $12 \mathrm{~Hz}$ is placed in the pump $(800 \mathrm{~nm})$ leg to provide a variable optical delay. The frequency doubled probe light $(400 \mathrm{~nm})$ is amplitude modulated at $1 \mathrm{MHz}$ to facilitate lock-in detection and the polarization plane is aligned with the bar axis.

Generation of SAPs using a suboptical wavelength absorption grating can be understood by considering the data presented in Fig 2. The change in reflectivity for a uniform, thick aluminum film is shown in Fig. 2 (a). The sharp change in the signal near $\mathrm{t}=0$ is caused by nonequilibrium heating and rapid cooling of the electron gas. This is followed by a slow decay caused by thermal diffusion. This portion of the signal is governed by a coupling of the temperature field to the sample reflectivity (thermoreflectance). The change in reflectivity for an absorption grating on a Si substrate is shown in Fig. 2 (b). In addition to the sharp increase in the signal near $t=0$ and the decaying thermal background, there is a high frequency ripple corresponding to SAP oscillations. 
This contribution to the signal is governed by a coupling between the strain field associated with the SAP oscillation and the sample reflectivity (photoelasticity).

The two signals shown in Fig 2 (c) and Fig. 2 (d) involve SAP oscillations in the Si and GaAs substrate respectively. The thermal background has been subtracted and the delay of the pump relative to the probe has been adjusted so as not to include $t=0$. The Fourier amplitude corresponding to the signals in the Si and GaAs substrate are shown in Fig. 2 (e) and Fig. 2 (f) respectively. The center frequency for the $\mathrm{Si}$ and GaAs samples is $22.4 \mathrm{GHz}$ and $13 \mathrm{GHz}$ respectively. These frequencies correspond closely to the frequency predicted using the Rayleigh velocity of the pristine substrate and the grating repeat length, $v=V_{R} / p(22.4 \mathrm{GHz}$ and $12.4 \mathrm{GHz}$ for $\mathrm{Si}$ and GaAs respectively). ${ }^{9}$

Generation of SAPs in this frequency range has exciting potential applications because the wavelength approaches the wavelength of phonons involved in heat pulse experiments. Furthermore, the wavelength is of the same order as length scales associated with quantum confined systems, thus enabling acoustically defined quantum structures. However, an important limitation involves the relatively small strain amplitude associated with surface acoustic phonons produced in this fashion (microstrain order). Increasing the strain amplitude of the SAP oscillation by increasing the pump fluence only works up to a certain point above which the grating is damaged due to excessive heat buildup. This limitation can be overcome by resonantly pumping the grating with multiple pulses. Since the grating can cool between pump pulses by conducting heat to the substrate, problems associated with thermal damage are effectively circumvented. Moreover, excitation with multiple pump pulses provides a means to coherently control SAP oscillations. This approach is similar to earlier studies involving coherent control of bulk optical and acoustic phonon oscillations ${ }^{10,11}$.

Multiple pump pulses can be derived using a variety of optical approaches. In this paper a Michelson interferometer setup was used to produce multiple pump beams. The schematic shown 
in Fig. 3 involves producing four pulses by combining two interferometers. It is important to note that the multiple pump pulses have orthogonal polarizations. For polarization sensitive generation schemes (like the present case involving a suboptical wavelength absorption grating) the polarizations can be made parallel by projecting the polarization vectors on a common axis using a polarizer. Aligning four pump beams proved to be a formidable challenge. As a consequence, only a single interferometer was used. A typical trace using two pump pulses is given in the inset of Fig. 3. Traces like this, that clearly reveal the $\mathrm{t}=0$ portion of both pulses, were used to adjust the timing between pump pulses.

The two pulse setup was used to coherently control SAP oscillations in both the Si and GaAs sample and revealed similar results. The Fourier amplitude of the SAP oscillations in the Si sample as a function of separation between the two pump pulses is shown in Fig. 4. The acoustic period of the SAP oscillation is approximately $45 \mathrm{ps}$. The Fourier amplitude is given over this delay period. The Fourier amplitude exhibits a sinusoidal dependence as a function of delay time, demonstrating coherent superposition of two phonon oscillations. The insets in Fig. 4 show the SAP oscillations for three different delays: constructive interference (i), partial constructive interference (ii) and destructive interference (iii). The thermal background has been subtracted and the delay between pump and probe pulses was adjusted so as not to include the $\mathrm{t}=0$ contribution from either pump pulse. Achieving total destructive interference demonstrates the precision with which the phonon oscillations can be coherently controlled.

\section{Conclusion}

In conclusion, coherent control of SAP oscillations produced by irradiating a sub-optical wavelength absorption grating with two pump pulses has been demonstrated. This approach has important potential applications involving coherent control of electronic properties of

piezoelectric quantum structures. Moreover, the potential for large strain amplitudes will enable fundamental studies of phonon-phonon interaction mediated by lattice anharmonicity. 


\section{Acknowledgement}

This work was sponsored by the U.S. Department of Energy, Office of Nuclear Energy, Advance

Fuel Cycle Initiative and a Laboratory Directed Research and Development program under DOE Idaho Operations Office Contract No. DE-AC07-05ID14517. 


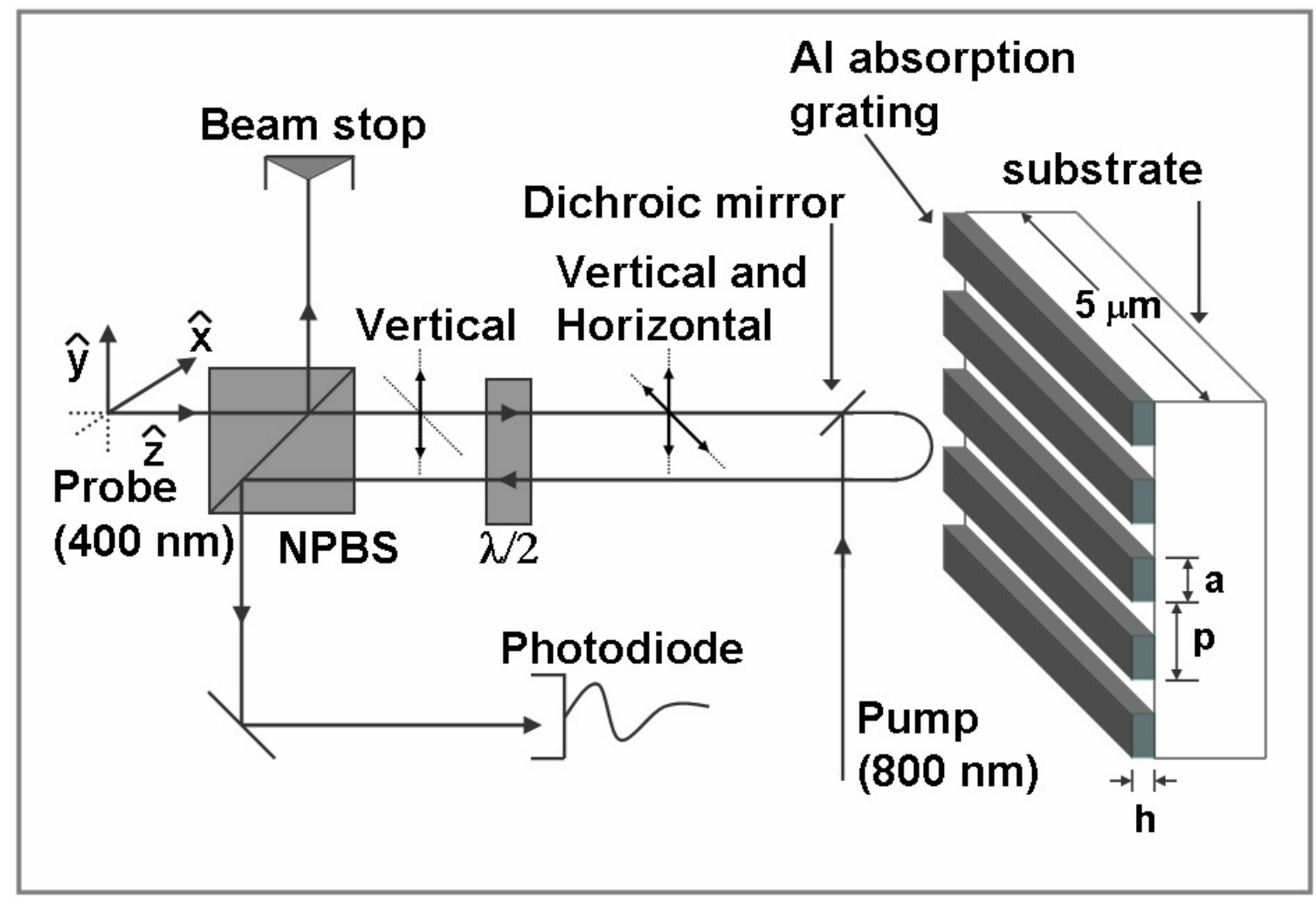

FIG. 1. The experimental setup. The polarization of the probe beam relative to the grating can be varied. The pump beam can be made collinear with the probe beam using a dichroic mirror. 

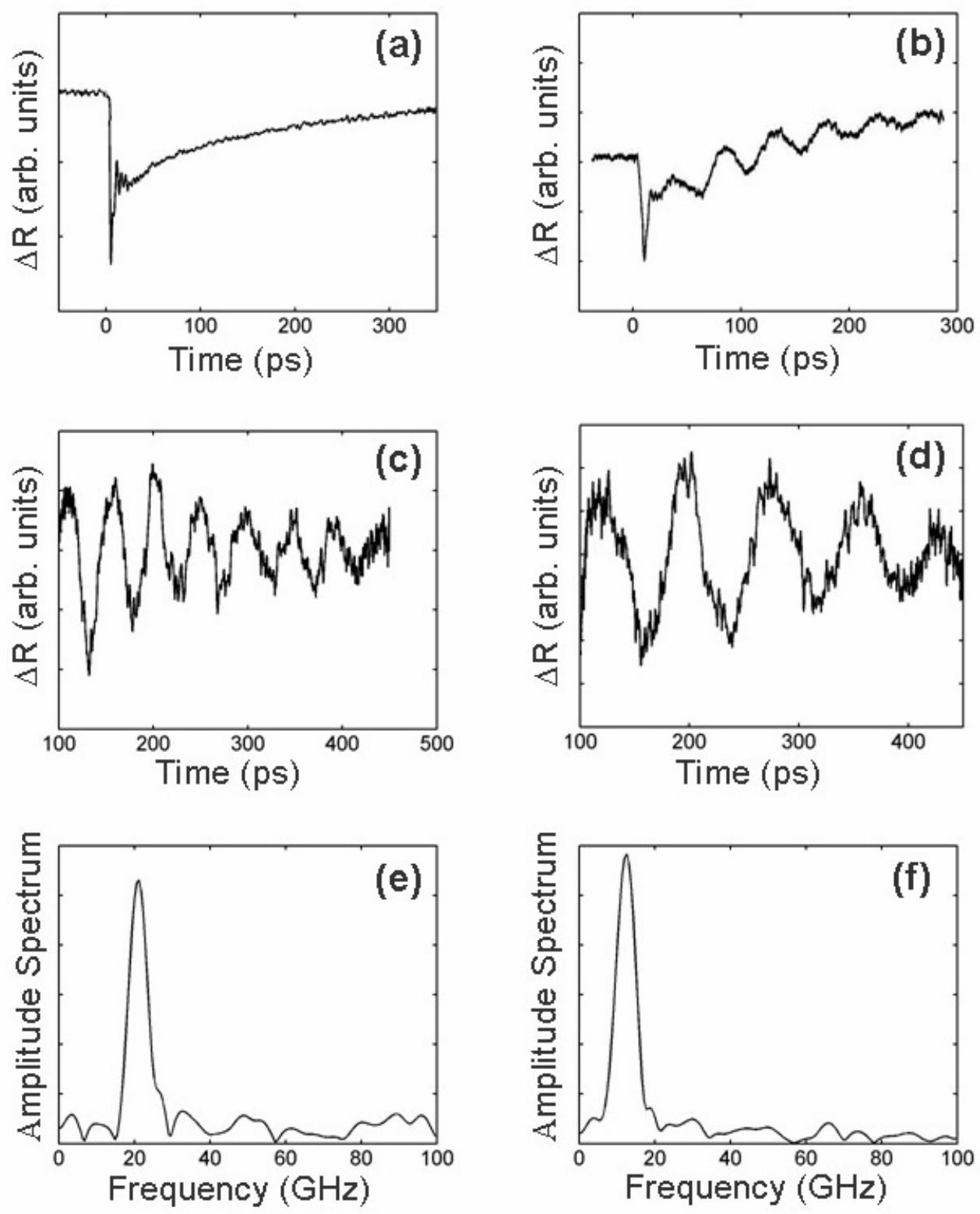

FIG. 2 (a) Change in reflectivity for a uniform thick Al film. (b) Change in reflectivity for an Al grating on a Si substrate. SAP oscillations in Si sample (c) and GaAs sample (d) with the thermal background subtracted. The Fourier amplitude spectrum corresponding to the Si sample (e) and the GaAs sample (f). 


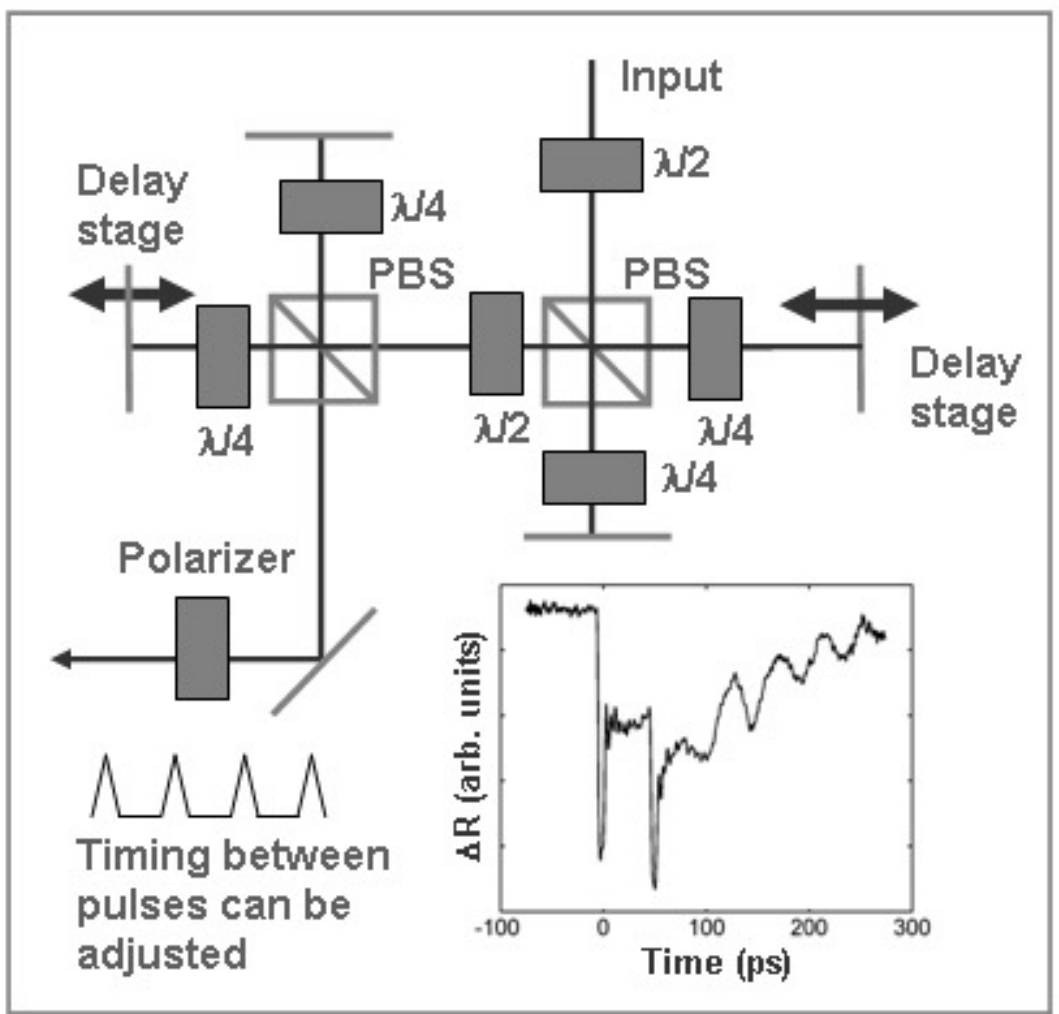

FIG. 3. A dual Michelson interferometer used to produce four pulses of equal amplitude. A polarizer can be used to project the orthogonal polarization vectors of the pulses on a common axis. Inset: Change in reflectivity clearly showing the arrival of the two pump pulses. 


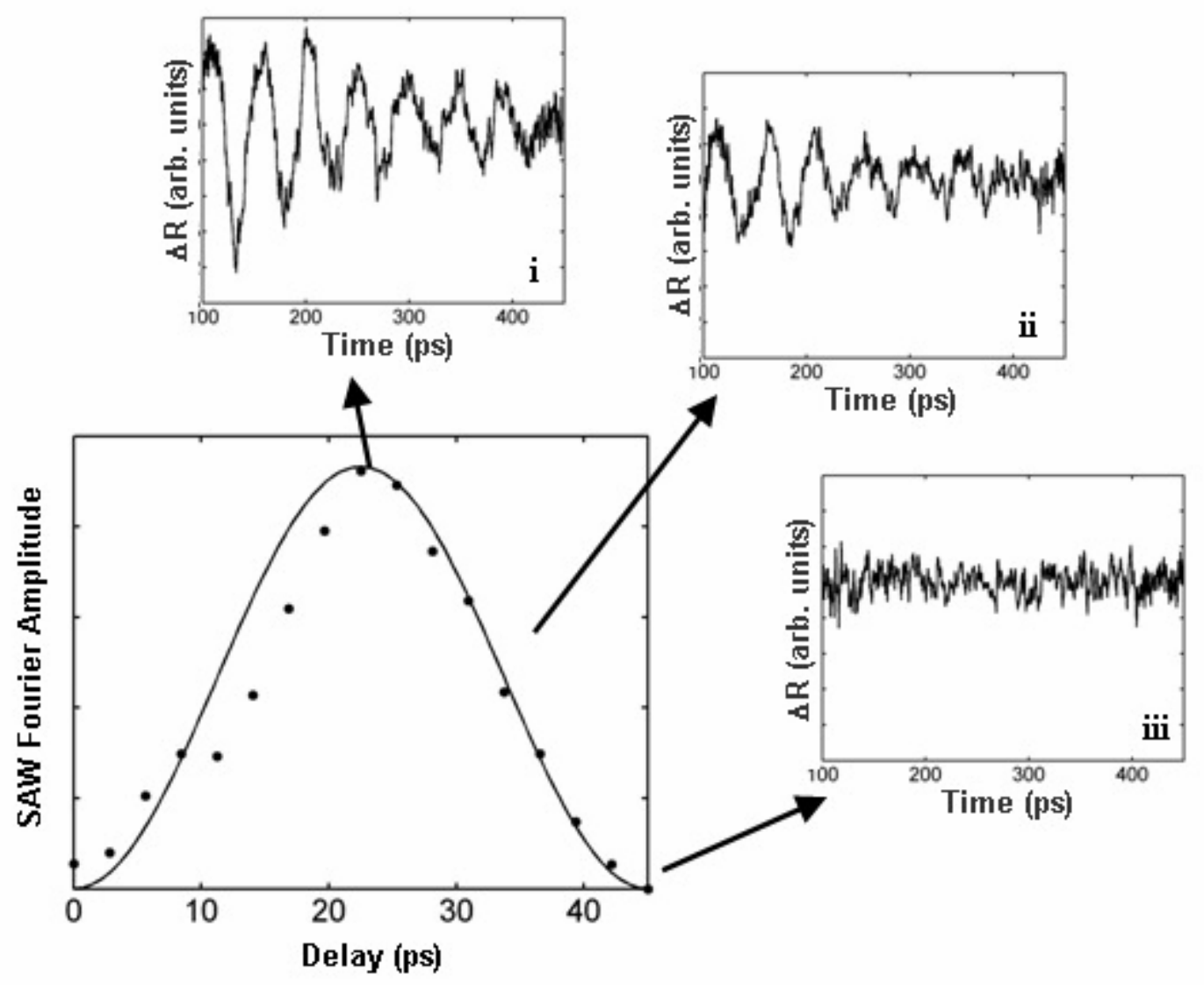

FIG. 4. The Fourier amplitude as a function of the delay between the two pump pulses. The sinusoidal dependence on delay time clearly demonstrates coherent superposition of two SAP oscillations. Insets: show the SAP oscillations at three different delays. 


\section{References}

1 D. G. Cahill, W. K. Ford, K. E. Goodson, G. D. Mahan, A. Majumdar, H. J. Maris, R. Merlin and S. R. Philpot, "Nanoscale thermal transport," J. Appl. Phys. 93, 793 (2003).

2 I.V. Kukushkin, J.H. Smet, L.Hoppel, U. Waizmann, M. Riek, Appl. Phys. Lett., 85, 4526 (2004).

3 A. M. Robinson, V. I. Talyanskii, M. Pepper, J. E. Cunningham, E. H. Linfield, D. A. Ritchie, Phys. Rev. B, 65, 45313 (2002).

4 J. A. H. Stotz, R. Hey, P. V. Santos, K. H. Ploog, Nature Mats. 4, 585 (2005).

5 C. Rocke, S. Zimmermann, A. Wixforth, J.P. Kotthaus, Phys. Rev. Lett., 78, 4099 (1997).

6 B. Bonello, A.Ajinou, V. Richard, Ph. Djemia, S.M. Cherif, J. Acoust. Soc. Am. 110, 1943 (2001).

7 D.H. Hurley, K.L. Telschow, Phys. Rev. B 66, 153301 (2002).

$8 \quad$ D.H. Hurley, Appl. Phys. Lett. 88, 191106 (2006).

9 In the present case, the pump spot size is approximately 2 microns and the repeat length is $220 \mathrm{~nm}$, thus the majority of the SAP energy is produced at the Brillouin zone boundary, $\lambda=\mathrm{p}$.

10 M. Hase, K. Mizoguch, H. Harima, S. Nakashima, M. Tani, K. Sakai, M. Hangyo, Appl. Phys. Lett. 69, 2474 (1996).

11 A. Bartels, T. Dekorsy, H. Kurz, K. Kohler, Appl. Phys. Lett. 72, 2844 (1998). 